\section{OPEN ACCESS}

Edited by:

Amine Benyamina,

Assistance Publique Hopitaux De

Paris, France

Reviewed by:

Raffaele Giorgetti,

Marche Polytechnic University, Italy

Angela Maria Sanna,

University of Cagliari, Italy

${ }^{*}$ Correspondence:

Alasdair M. Barr

al.barr@ubc.ca

Specialty section: This article was submitted to

Addictive Disorders,

a section of the journal

Frontiers in Psychiatry

Received: 09 December 2020

Accepted: 18 February 2021

Published: 12 March 2021

Citation:

Eadie L, Lo LA, Christiansen A, Brubacher JR, Barr AM, Panenka WJ and MacCallum CA (2021) Duration of

Neurocognitive Impairment With

Medical Cannabis Use: A Scoping

Review. Front. Psychiatry 12:638962.

doi: 10.3389/fpsyt.2021.638962

\title{
Duration of Neurocognitive Impairment With Medical Cannabis Use: A Scoping Review
}

\author{
Lauren Eadie ${ }^{1}$, Lindsay A. Lo ${ }^{2}$, April Christiansen ${ }^{3}$, Jeffrey R. Brubacher ${ }^{4}$, \\ Alasdair M. Barr ${ }^{5,6 *}$, William J. Panenka ${ }^{6,7,8}$ and Caroline A. MacCallum ${ }^{1}$ \\ ${ }^{1}$ Department of Medicine, Faculty of Medicine, University of British Columbia, Vancouver, BC, Canada, ${ }^{2}$ Department of \\ Psychology, Queens University, Kingston, ON, Canada, ${ }^{3}$ Centre for Neuroscience Studies, Queens University, Kingston, ON, \\ Canada, ${ }^{4}$ Department of Emergency Medicine, Faculty of Medicine, University of British Columbia, Vancouver, BC, Canada, \\ ${ }^{5}$ Department of Anesthesiology, Pharmacology \& Therapeutics, Faculty of Medicine, University of British Columbia, \\ Vancouver, BC, Canada, ${ }^{6}$ British Columbia Mental Health and Substance Use Services Research Institute, Vancouver, BC \\ Canada, ${ }^{7}$ Department of Psychiatry, Faculty of Medicine, University of British Columbia, Vancouver, BC, Canada, ${ }^{8}$ British \\ Columbia Provincial Neuropsychiatry Program, Vancouver, BC, Canada
}

While the recreational use of cannabis has well-established dose-dependent effects on neurocognitive and psychomotor functioning, there is little consensus on the degree and duration of impairment typically seen with medical marijuana use. Compared to recreational cannabis users, medical cannabis patients have distinct characteristics that may modify the presence and extent of impairment. The goal of this review was to determine the duration of acute neurocognitive impairment associated with medical cannabis use, and to identify differences between medical cannabis patients and recreational users. These findings are used to gain insight on how medical professionals can best advise medical cannabis patients with regards to automobile driving or safety-sensitive tasks at work. A systematic electronic search for English language randomized controlled trials (RCTs), clinical trials and systematic reviews (in order to capture any potentially missed RCTs) between 2000 and 2019 was conducted through Ovid MEDLINE and EMBASE electronic databases using MeSH terms. Articles were limited to medical cannabis patients using cannabis for chronic non-cancer pain or spasticity. After screening titles and abstracts, 37 relevant studies were subjected to full-text review. Overall, seven controlled trials met the inclusion/exclusion criteria and were included in the qualitative synthesis: six RCTs and one observational clinical trial. Neurocognitive testing varied significantly between all studies, including the specific tests administered and the timing of assessments post-cannabis consumption. In general, cognitive performance declined mostly in a THC dose-dependent manner, with steady resolution of impairment in the hours following THC administration. Doses of THC were lower than those typically reported in recreational cannabis studies. In all the studies, there was no difference between any of the THC groups and placebo on any neurocognitive measure after $4 \mathrm{~h}$ of recovery. 
Variability in the dose-dependent relationship raises the consideration that there are other important factors contributing to the duration of neurocognitive impairment besides the dose of THC ingested. These modifiable and non-modifiable factors are individually discussed.

Keywords: cannabinoids, medical cannabis, tetrahydrocannabinol, cannabidiol, pain, impairment, intoxication, cognition

\section{INTRODUCTION}

The legalization and decriminalization of cannabis in multiple countries and states has contributed to a wealth of research on the potential therapeutic benefits of cannabis-based medicines (1-5). In 2014, cannabinoids were deemed appropriate as third-line treatment for neuropathic pain by the Canadian Pain Society (6). Cannabis has also been investigated as an adjuvant in refractory chronic non-cancer pain and in harm-reduction approaches for those tapering off high-dose opioid medications, with promising preliminary findings (711). As the indications for cannabis expand beyond neuropathic pain, seizures and multiple sclerosis (MS)-related spasticity, it is necessary to assess the risks associated with medicinal cannabis use, especially among those who regularly ingest THCcontaining compounds.

Research on the effects of cannabis on humans has largely focused on recreational use, with smoking as the most common route of administration. This early work found strong associations between the dose of THC inhaled and resulting acute cognitive impairment (12). Specifically, THC and other cannabinoid receptor $1\left(\mathrm{CB}_{1}\right)$ agonists acutely impair psychomotor and neurocognitive domains including attention, manual dexterity, coordination, and reaction time, as $\mathrm{CB}_{1}$ receptors are neuroanatomically expressed in regions responsible for cognitive and motor control $(13,14)$. Therefore, THC dose-dependently disrupts important cognitive and psychomotor functions needed for safety-sensitive work, including driving motorized vehicles $(15,16)$.

There is currently no standardized definition of impairment associated with medical cannabis use in the literature and therefore, no general consensus on how to measure or define this impairment. Unlike with alcohol, where blood alcohol levels directly correlate with the degree of intoxication, the relationship between cannabinoid and neurocognitive or functional impairment remains undetermined. While evidence supports a positive relationship between THC dose and impairment, an accurate blood concentration range has not been determined (17). Some studies have suggested THC blood concentrations between 2 and $5 \mathrm{ng} / \mathrm{ml}$ are associated with impairment (18-20). However, these measures do not consistently correlate with impairment across individuals $(17,21)$. This is likely due to the complex nature of THC pharmacokinetics and metabolism $(17,20)$ which is strongly impacted by individual factors such as genetics and tolerance to THC.
The two main metabolites of THC include the primary psychoactive metabolite "11-hydroxytetrahydrocannabinol" (11-OH-THC) and the second metabolite "11-nor-9-carboxytetrahydrocannabinol" (THC-COOH) (22). The latter is a non-psychoactive and non-intoxicating cannabis metabolite which is usually eliminated from the body within 5 days of consumption primarily via feces and urine (23). From recreational cannabis studies, the detectable half-life of THC$\mathrm{COOH}$ is much longer than for THC and 11-OH-THC. For infrequent cannabis users the half-life of THC-COOH is around 1.3 days, while for frequent users it is in the range of 5-13 days (24). The practical implication for medical cannabis patients is that they would likely test positive for cannabis on urine drug tests (which typically detect THC-COOH) days after last using THC (22). As THC-COOH is not psychoactive, its prolonged presence in frequent users is not a valid biomarker of impairment.

There is evidence that medical cannabis patients who use THC regularly develop tolerance to many of the impairing effects of THC (25). Tolerance has also been found with recreational cannabis use, with experimental studies demonstrating that frequent recreational cannabis users, with use more than four times per week, developed psychological and behavioral tolerance, and showed no significant impairment in neurocognitive function or motor side effects compared to infrequent users at the same dose of THC $(26,27)$. Other research demonstrates that tolerance is incomplete, and people who use cannabis regularly still demonstrate some impairment, albeit blunted, after acute use (28).

Determining the duration of potential THC impairment, and what THC dose a medical cannabis patient should take to minimize neurocognitive impairment, proves to be challenging. There are some unique considerations when studying impairment in medical cannabis patients, defined here as someone who uses cannabis under the guidance of a medical practitioner, compared to recreational cannabis users. Medical cannabis patients often use THC to manage symptoms for a variety of conditions including chronic pain, insomnia, PTSD, autoimmune conditions, and neurological disorders, that induce a certain level of neurocognitive impairment by themselves. By treating these symptoms, their neurocognitive and psychomotor functioning may actually improve. Medical cannabis patients also have different patterns of use, including a more consistent and standardized dosing schedule, along with different expectations and goals (29). They often consume cannabis orally, which lengthens the time until onset and the duration of effect after use, and choose use chemovars high in cannabidiol (CBD), which 
is non-impairing (30). If medical cannabis patients are starting THC, most start with low-dose THC products, with doses titrated to obtain symptomatic relief while purposely avoiding impairing side-effects.

The aim of the present scoping review was to identify and summarize studies that investigate the duration and degree of acute neurocognitive impairment with medical cannabis use, and to compare this literature with the body of research on neurocognitive impairment in recreational cannabis users (3135). Impairment, for the purposes of this review, is considered as disruption in neurocognitive and motor tasks that, if present, could potentially cause harm to the subject or others (e.g., driving or workplace safety). To investigate this critical question, we performed a scoping review of clinical trials that used standardized neurocognitive and psychomotor tests to study medical cannabis patients preceding and following acute THC administration. These findings are then compared to similar research involving recreational cannabis users to explore unique features of the medical cannabis patient population. We conclude by proposing a provisional standardized neurocognitive and psychomotor assessment battery for studying acute THC impairment in medical cannabis patients, and by discussing how medical professionals can best advise patients with regards to safety-sensitive work, including driving.

\section{MATERIALS AND METHODS}

This study is a scoping review and qualitative analysis of the literature on impairment in medical cannabis patients. A systematic electronic search for English language randomized controlled trials (RCTs), clinical trials and systematic reviews (in order to capture any potentially missed RCTs) between 2000 and 2019 was conducted through Ovid MEDLINE and EMBASE electronic databases using the following MeSH terms: (exp Cannabinoids/ OR cannabi* OR dronabinol OR marijuana OR tetrahydrocannabinol OR THC OR Sativex) AND (chronic non? cancer pain OR Chronic Pain/OR muscle spasticity/OR spasticity) AND (impair* OR cognition OR intoxication OR reaction time OR coordination OR neurocognitive OR psychomotor). This search strategy was developed with the assistance of a medical librarian, and was conducted as we have previously reported on prior studies of drug-associated psychological effects (36-38).

Titles and abstracts were reviewed and obviously irrelevant studies were excluded. Full text of the remaining studies was reviewed to determine eligibility. The review was performed by a single investigator. Input from a second investigator was sought as required. The current focus was on medical cannabis patients using cannabis for chronic non-cancer pain or spasticity. Studies were included if they documented dose, product type and method of THC administration in addition to having formal objective neurocognitive or psychomotor baseline and acute post-THC assessments. See Table 1 for PICO statement. Abstracts were analyzed for inclusion based on PRISMA criteria. Studies were excluded if they focused solely on recreational cannabis use, did not have any objective neurocognitive or psychomotor testing, or
TABLE 1 | PICOS breakdown of study eligibility criteria.

\begin{tabular}{|c|c|}
\hline $\begin{array}{l}\text { P (Problem or } \\
\text { Patient or } \\
\text { Population) }\end{array}$ & $\begin{array}{l}\text { Adults living with chronic, non-cancer pain (pain of } \\
>3 \text {-month duration) and/or spasticity. }\end{array}$ \\
\hline (Intervention/indicator) & Medical cannabis use or cannabinoid-based medicines. \\
\hline C (Comparison) & $\begin{array}{l}\text { Chronic pain/spasticity controls (without cannabis use). } \\
\text { Studies without comparators will also be included. }\end{array}$ \\
\hline $\begin{array}{l}\text { O (Outcome of } \\
\text { interest) }\end{array}$ & $\begin{array}{l}\text { Duration of acute neurocognitive and psychomotor } \\
\text { impairment using objective standardized measures }\end{array}$ \\
\hline $\begin{array}{l}\text { S (Study types } \\
\text { selected) }\end{array}$ & $\begin{array}{l}\text { Randomized controlled trials and other clinical trials will } \\
\text { be included. }\end{array}$ \\
\hline
\end{tabular}

TABLE 2 | Inclusion and exclusion criteria (for medical cannabis patients using cannabis for chronic non-cancer pain or spasticity).

Inclusion criteria

Cannabis and the management of chronic non-cancer pain and/or spasticity

Efficacy, tolerability, and safety studies on the use of medical cannabis for chronic non-cancer pain and/or spasticity

Exclusion criteria

Studies in a language other than English
Studies published before 2000
Studies which focus on recreational cannabis use
Studies focusing on cannabis use disorder
Studies without any formal and objective/reproducible
neurocognitive testing
Studies investigating the non-acute use of cannabis (for
example, impairment after using daily THC for 1 month,
instead of 1 h-post consumption)
Studies on animals

if the testing was done following subacute exposure, such as after weeks or months of daily THC exposure (Table 2).

Systematic reviews on medical cannabis use were also evaluated. Three additional RCTs that met the inclusion criteria were found in the references of these systematic reviews and were added to the analysis. One newly published observational clinical trial discovered through exert recommendation was added to the final analysis that was not found in our original electronic search. A database was not created from our review.

Data extracted from the investigated studies included the type of study completed, the number of participants, the participant characteristics, such as their medical condition causing pain or spasticity and their previous experience with cannabis, (or presumed THC tolerance), the THC concentrations assessed, the THC dosing intervals, the neurocognitive tests utilized, the timing of the neurocognitive testing intervals and the results of these neurocognitive tests for each THC dose and timing interval. The data drawn from the included studies was interpreted and summarized to make a preliminary recommendation on the duration of neurocognitive and motor impairments in medical cannabis users. 


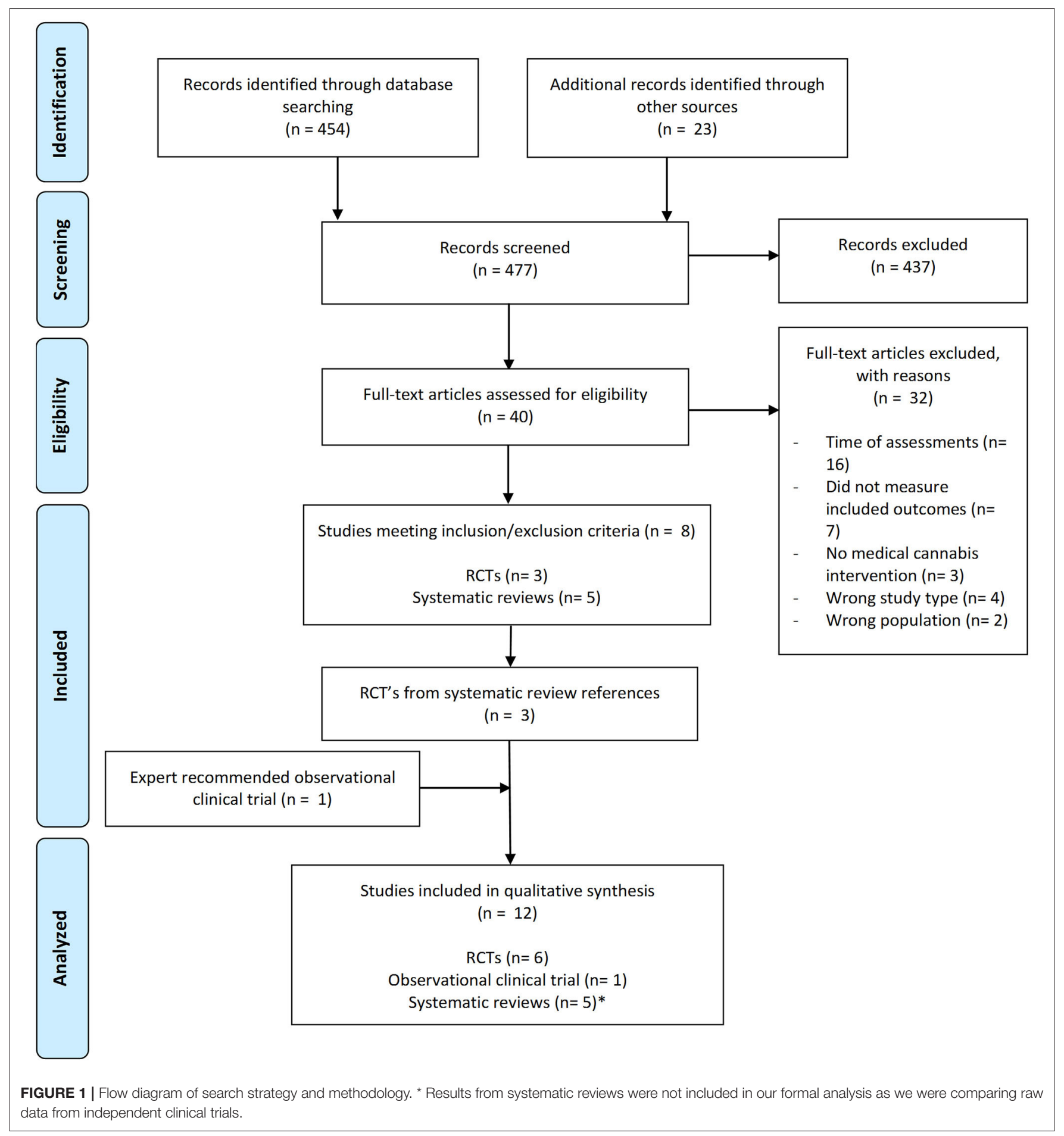

\section{RESULTS}

We identified 454 potentially eligible publications from the search strategy and twenty other potential articles from other resources. After screening titles and abstracts, 37 relevant studies were subjected to full-text review. One review article analyzed contained three additional RCTs which were independently reviewed for a total of 40 relevant studies reviewed. 32 studies were excluded for the following reasons: they measured subacute impairment of THC (days to weeks after ingestion), they did not have formal neurocognitive testing, there was no formal medical THC intervention completed, the study was not interventional, or they did not study adults living with chronic, non-cancer pain and/or spasticity. Eight studies met 
our final criteria, five systematic reviews and three RCT's. From the systematic reviews, three RCT's were extracted for analysis. One newly published observational clinical trial discovered through expert recommendation was added that was not found in our original search. Overall, seven controlled trials met the inclusion/exclusion criteria and were included in the qualitative synthesis: six RCTs and one observational clinical trial. A flow diagram of our search strategy summarizes our methodology (Figure 1).

\section{Study Characteristics}

Study characteristics for the six RCTs and one observational trial are summarized in Table 3 (39-45). A total of 234 medical cannabis patients were included in these studies: 175 patients with neuropathic pain, 37 patients with MS-associated spasticity and 22 patients prescribed medical cannabis pre-dominantly for chronic pain, anxiety or depression.

Route of cannabis administration varied: two studies required patients to smoke cannabis, three used vaporized cannabis, one allowed for smoking or vaporizing, and one study used sublingual THC, CBD, or THC: CBD spray. All three vaporization studies utilized the Foltin Puff Procedure, where participants are verbally signaled to "hold the vaporizer bag with one hand and put the vaporizer bag mouthpiece in their mouth" (30s), "get ready" ( $5 \mathrm{~s})$, "inhale" ( $5 \mathrm{~s})$, "hold vapor in lungs" (10 s), "exhale and wait" before repeating the puff cycle $(40 \mathrm{~s})(39,43,44)$.

Four of the seven studies required participants to abstain from non-study cannabis use for at least 30 days prior to the start of the study $(39,40,44)$. Two of the four verified abstinence through negative urine drug screens $(39,41)$. Several of the studies allowed medical cannabis use prior to the study initiation $(42,45)$, with less than half of the participants from one study reporting regular cannabis use (43).

There were a variety of testing protocols, with significant variability on the timing of THC or placebo administration and when the neurocognitive testing was completed. Some studies performed a single THC administration (39, 41, 45), where others had cumulative inhalation procedures (40, 42-44). Neurocognitive testing was either singular or repeated, with the most complete testing at baseline and every $30 \mathrm{~min}$ for $3 \mathrm{~h}$ total after THC ingestion (39).

\section{Summary of Findings}

Neurocognitive testing varied significantly between all studies, including the specific tests administered and the timing of assessments post-cannabis consumption. Table 3 provides findings from individual studies, while Table 4 provides details about the neurocognitive tests administered and the cognitive modalities examined with each test.

Two of the three studies using the Trails Making Test to assess visual attention and processing speed with switching tasks did not find significant differences between THC groups compared to placebo except for at two timepoints $(39,43)$. In one study, the low-dose THC group took longer than the high-dose THC group on the Trails A at $420 \mathrm{~min}$, immediately after the second THC dosing interval (43). The second study found the high dose group took longer compared to placebo on the Trails B at $120 \mathrm{~min}$ post-dose (39). The third study assessing the Trails Making Test did not report their quantitative results in their findings (42).

Of the three studies using the Paced Auditory Serial Attention Test for auditory processing speed and working memory (39, 41,43 ), one study found no significant differences between THC groups and placebo at any timepoint, but the high-dose THC group performed better than the low-dose THC group at $420 \mathrm{~min}$ (43). In the second study, the high- and medium-dose THC groups had worse performance than placebo at $15 \mathrm{~min}$ postinhalation, but there was no difference in performance between low, medium, or high dose THC groups compared to placebo at the following 60-, 120- or 240-min post-inhalation testing (39). In the final study, the THC group had worse performance compared to placebo at $45 \mathrm{~min}$ post-inhalation with no further testing after this timepoint (41).

Results were mixed between the three studies using the Grooved Pegboard Test (GPT) $(40,43,44)$ to assess dexterity and fine motor control. All three studies used cumulative cannabis inhalation protocols. One study found no significant effects across active doses compared to placebo on the dominanthand GPT but observed decreased performance on the nondominant GPT in the high-dose THC group compared to placebo. This occurred 1-h after the second THC dosing session and resolved after an additional $60 \mathrm{~min}$ (43). In the second study, the low-dose THC group had worse performance than the medium-dose THC and the placebo group on the dominanthand GPT at $60 \mathrm{~min}$, (immediately after the first dosing session), and $240 \mathrm{~min}$, (60 min after the second dosing session) (44). This same study found that both the low-dose and medium-dose THC groups had decreased performance on the non-dominant GPT at the 120- and 180-min (60 min after first dosing session and immediately after the second dosing session) (44). There was no difference in performance between placebo and either THC group at the 300 -min mark, $3 \mathrm{~h}$ after the last scheduled inhalation (44). The final study found a decrease in overall performance in the high-dose THC group compared to placebo on the dominanthand GPT, but no difference between the low-dose THC group and placebo. In the non-dominant hand GPT, this study found that both THC groups had decreased performance compared to placebo. The study measured maximal recovery $2 \mathrm{~h}$ after the last inhalation session at $180 \mathrm{~min}$ where low-dose and high-dose THC groups had significant improvement on the GPT compared to their previous scores (40).

All three studies that administered the Hopkins Verbal Learning Test and Delayed Learning Test to assess learning, immediate and delayed recall found THC dose-dependent impairment on learning and recall compared to placebo (40, 43, 44). For two studies, performance following higher THC doses was worse than for lower doses of THC, which in turn, were worse than placebo $(40,44)$. Notably, one study found poor performance on this test even in the placebo group, hypothesized to be due to their underlying neuropathic pain condition (40). The second study found recovery of these differences $2 \mathrm{~h}$ after the last inhaled THC session (44). The final study found no difference in test scores between the low-dose THC group and placebo. In this study, the high-dose THC group had fewer true-positive responses and more false positives compared to 
TABLE 3 | Study characteristics and results.

\begin{tabular}{|c|c|c|c|c|c|}
\hline Study & Population & Intervention & Cannabis use & Outcome & Results \\
\hline $\begin{array}{l}\text { Wallace et al. (39) } \\
\text { Randomized, } \\
\text { double-blind, } \\
\text { placebo-controlled } \\
\text { crossover study }\end{array}$ & $\begin{array}{l}\text { Painful Diabetic } \\
\text { Neuropathy } \\
16 \text { participants }\end{array}$ & $\begin{array}{l}\text { Placebo, 1, 4, and } 7 \% \text { THC } \\
\text { vaporized } \\
4 \text { inhalations using the Foltin Puff } \\
\text { Procedure in one single dosing } \\
\text { session (equaling } 0,4,16 \text {, or } \\
28 \mathrm{mg} \text { THC) }\end{array}$ & $\begin{array}{l}\text { No use of cannabis in } \\
\text { past } 30 \text { days prior to } \\
\text { study tested by urine } \\
\text { drug screen }\end{array}$ & $\begin{array}{l}\text { Trail Making Test } \\
\text { Paced Auditory Serial Attention } \\
\text { Test } \\
\text { Testing at 5-min, 30-min and } \\
\text { every } 30-\text { min for } 3 \mathrm{~h} \text {. Final } \\
\text { measurement at 240-min. }\end{array}$ & $\begin{array}{l}\text { Decline in neurocognitive performance with THC exposure } \\
\text { which was dose dependent and improved with time. No } \\
\text { difference in any groups at } 240 \text {-min post-inhalation (4-h). } \\
\text { Trails: } 7 \% \text { THC group took longer compared to placebo on } \\
\text { Trails B at } 120 \text {-min. No difference between } 1 \text { and } 4 \% \text { THC } \\
\text { groups and placebo } \\
\text { Paced Auditory Serial Addition Test: } 7 \% \text { THC and } 4 \% \text { THC } \\
\text { groups had worse performance than placebo at } 15 \text {-min } \\
\text { post-THC dose. There was no difference in performance } \\
\text { between 1, } 4 \text {, or } 7 \% \text { THC groups compared to placebo at the } \\
\text { following } 60-, 120-\text {, or } 240 \text {-min testing. }\end{array}$ \\
\hline $\begin{array}{l}\text { Wilsey et al. (40) } \\
\text { Double-blind, } \\
\text { placebo-controlled, } \\
\text { crossover study }\end{array}$ & $\begin{array}{l}\text { Central and Peripheral } \\
\text { Neuropathic Pain } \\
38 \text { participants }\end{array}$ & $\begin{array}{l}\text { Placebo vs. } 3.5 \% \text { THC vs. } 7 \% \\
\text { THC smoked } \\
2 \text { inhalations at } 60-\text { min, } 3 \\
\text { inhalations at } 120-\text { min, and } 4 \\
\text { inhalations at } 180-\text { min for a total } \\
\text { of } 9 \text { cumulative inhalations (total } \\
\text { estimate: } 19 \mathrm{mg} \text { THC low dose, } \\
34 \mathrm{mg} \text { THC high dose) }\end{array}$ & $\begin{array}{l}\text { All had previous } \\
\text { cannabis exposure } \\
\text { No cannabis } 30 \text { days } \\
\text { prior to study }\end{array}$ & $\begin{array}{l}\text { Digit Symbol Test } \\
\text { Hopkins Verbal Learning Test } \\
\text { and Delayed Learning } \\
\text { Grooved Pegboard Dominant } \\
\text { and Non-Dominant tests } \\
\text { Testing completed at baseline, } \\
60-\text { mins (after } 2 \text { puffs), 120-min } \\
\text { (after } 3 \text { puffs), 180-mins (after } 4 \\
\text { puffs), 240-min (after } \\
\text { 1-h recovery). }\end{array}$ & $\begin{array}{l}\text { Modest decline in cognitive performance with THC use, most } \\
\text { significant in the 7\% THC group. } 76 \% \text { of participants had } \\
\text { cognitive impairment at baseline. } \\
\text { Digit Symbol Test: no significant dose-effect differences } \\
\text { Hopkins: } 7 \% \text { THC group had worse performed than the 3.5\% } \\
\text { THC group which performed worse than placebo. Poor } \\
\text { performance even in placebo group } \\
\text { Dominant-hand Pegboard: } 7 \% \text { THC group performed worse } \\
\text { than placebo. No difference in performance between the } \\
\text { 3.5\% THC group and placebo. } \\
\text { Non-dominant hand pegboard: Both THC groups had } \\
\text { decreased performance compared to placebo. 2-h after the } \\
\text { last inhalation session, both THC groups had significant } \\
\text { improvement compared to their previous scores }\end{array}$ \\
\hline $\begin{array}{l}\text { Corey-Bloom et al. (41) } \\
\text { Randomized } \\
\text { placebo-controlled trial }\end{array}$ & $\begin{array}{l}\text { Multiple Sclerosis } \\
\text { Spasticity } \\
37 \text { participants }\end{array}$ & $\begin{array}{l}\text { Placebo vs. } 4 \% \text { THC smoked } \\
4 \text { inhalations of } 4 \% \text { THC smoked } \\
\text { in one dosing session } \\
(\sim 16 \mathrm{mg} \text { THC) }\end{array}$ & $\begin{array}{l}\text { Cannabis naïve or } \\
\text { negative toxicological } \\
\text { screen for } \mathrm{THC} \text { at } \\
\text { study initiation }\end{array}$ & $\begin{array}{l}\text { Timed walk score } \\
\text { Paced Auditory Serial Addition } \\
\text { Test } \\
\text { Baseline and } \\
\text { 45-min post-treatment }\end{array}$ & $\begin{array}{l}\text { Timed walk: no difference } \\
\text { Paced Auditory Serial Addition Test: } 4 \% \text { THC group had } \\
\text { worse performance compared to placebo at } 45 \text {-min. } \\
\text { There was no neurocognitive testing beyond } 45 \text {-min. }\end{array}$ \\
\hline $\begin{array}{l}\text { Notcutt et al. (42) } \\
\text { Prospective, } \\
\text { randomized, } \\
\text { double-blind, } \\
\text { placebo-controlled } \\
\text { crossover study }\end{array}$ & $\begin{array}{l}\text { Chronic mostly } \\
\text { neuropathic pain } \\
34 \text { participants }\end{array}$ & $\begin{array}{l}\text { Sublingual Spray } \\
2.5 \mathrm{mg} \text { THC vs. } 2.5 \mathrm{mg} \text { CBD vs. } \\
2.5 \mathrm{mg} \text { THC and } 2.5 \mathrm{mg} \text { CBD } \\
\text { One spray every } 15-30 \mathrm{~min} \text { and } \\
\text { individually stopped further } \\
\text { dosing after response was } \\
\text { achieved } \\
\text { Total intake: } 2-8 \text { sprays over a } \\
\text { 4-h period ( 5-20 mg THC) }\end{array}$ & $\begin{array}{l}\text { Excluded if significant } \\
\text { past or current } \\
\text { recreational cannabis } \\
\text { use, okay if medical } \\
\text { cannabis use }\end{array}$ & $\begin{array}{l}\text { Trail Making Tests A \& B } \\
\text { Adult Memory and Information } \\
\text { Processing Battery } \\
\text { Baseline and } 3-h \text { post-dose }\end{array}$ & $\begin{array}{l}\text { Equivocal results, requiring a more detailed analysis than the } \\
\text { study planned. Testing often improved after the initiation of } \\
\text { cannabis-based medicine. }\end{array}$ \\
\hline
\end{tabular}


Wilsey et al. (43)

Crossover, randomize

placebo-controlled

Patients with refractory

human

laboratory experiment neuropathic pain who have disease or injury to their spinal cord

48 participants

Placebo vs. $2.9 \%$ vs. $6.7 \%$ THC vaporized

4 puffs using the Foltin Puff Procedure at 60-min with a second dosing session at

240-min of 4-8 puffs

(flexible dosing schedule: the

participant chooses their second dose between 4-8 puffs)

Central or peripheral neuropathic pain (Refractory) 39 participants
Randomized

double-blind placebo

controlled

cross-over trial

17/42 participants used Wechsler Adult Intelligence Scale cannabis regularly Digit Symbol Test Some were cannabis naiive or ex-users

Trail Making Test

Grooved Pegboard Test Paced Auditory Serial Addition

Hopkins Verbal Learning Test Revised with 20-min delay Neurocognitive testing every hour (with variations to prevent learning)

Placebo vs. $1.29 \%$, vs. $3.53 \%$ THC vaporized 4 puffs at using the Foltin Puff Procedure at 60 -mins with a second dosing session at 180 -min of 4-8 more puffs (flexible dosing schedule: the participant chooses their second dose between 4 and 8 puffs)
All had previous cannabis exposure No cannabis 30 days prior to study
Wechsler Adult Intelligence Scale Digit Symbol Test

Hopkins Verbal Learning Test Revised

Grooved Pegboard Test

Baseline, 60-, 120-, 180-, 240-,

and 300-min after administration of $\mathrm{THC}$
Measurement of neurocognitive performance proved technically challenging due to the various disabilities in the population studied. THC showed dose-dependent neurocognitive impairment with resolution $2 \mathrm{~h}$ after inhalation of THC.

Dominant-hand Pegboard: no significant dose-effect differences

Non-Dominant Hand Pegboard: 6.7\% THC group performed worse compared to placebo 1 -h after the 2nd THC dosing session. Resolved 1-h later

Digit Symbol Test: no significant dose-effect differences, with all groups improving scores over time, consistent with practice effects

Trail Making Test-A: 2.9\% THC group took longer than the $6.7 \%$ THC group on the Trails A at 420 min, immediately after the 2nd THC dosing interval

Hopkins: no difference in test scores between the 2.9\% THC group and placebo. 6.7\% THC group had less true-positive and more false-positive responses compared to placebo. Resolved 2-h after the 2nd dosing session

Paced Auditory Serial Addition Test: no significant differences between THC groups and placebo at any timepoint. 6.7\%

THC group performed better than the $2.9 \%$ THC group at $420 \mathrm{~min}, 3$-h after the 2 nd dosing interval

THC produced a short duration of neurocognitive impairment. No difference in performance between $\mathrm{THC}$ and placebo 2-h after the last dosing session

Digit Symbol Test: 1.29 and 3.53\% THC groups had worse performance at 60-min, (after 1st inhalation) and 180-min, (after the 2nd inhalation) compared to placebo. No difference in either THC group and placebo at 120- and 240-min (1-h after each dose)

Dominant Hand Pegboard: 1.29\% THC group had worse performance than the $3.53 \% \mathrm{THC}$ and the placebo group at 60-min (after 1st inhalation) and 240-min, (60-min after 2nd inhalation) which resolved 60-min later

Non-dominant Hand Pegboard: $1.29 \%$ THC and 3.53\% THC groups had decreased performance at 120-min (60-min after 1st inhalation) and 180-min (after 2nd inhalation) which resolved 60-min later

Hopkins: performance following higher THC doses was worse than for lower doses of THC, which in turn, were worse than placebo. There was recovery of these differences $2-h$ after the last THC inhalation session. 




\section{DISCUSSION}

This scoping review provides evidence that cognitive performance in medical cannabis patients acutely declines after THC use, with steady resolution of impairment in the hours following THC administration. The degree of impairment is predominantly dose-dependent; higher doses of THC are generally more impairing than the lower doses. The duration of neurocognitive impairment varied between studies, partly due the heterogeneity in study designs. Nonetheless, there was no difference on any neurocognitive test between placebo and the active THC groups at 4 -h of recovery, irrespective 
TABLE 4 | Neurocognitive tests and cognitive domains.

\begin{tabular}{|c|c|}
\hline Neurocognitive test & Neurocognitive correlate assessed \\
\hline Paced Auditory Serial Attention Test & Auditory information processing speed and working memory \\
\hline Wechsler Adult Intelligence Scale Digit Symbol Test & Concentration, psychomotor speed, and graphomotor abilities \\
\hline Trail Making Test $A$ and $B$ & Processing speed, visual attention, and task-switching \\
\hline Grooved Pegboard Test (Dominant and Non-Dominant) & Fine motor coordination and speed \\
\hline Hopkins Verbal Learning Test Revised with 20-min delay & $\begin{array}{l}\text { Learning/ability to retain, reproduce, and recognize information after a } 20 \text { min delay. Immediate and delayed } \\
\text { recall of verbal information }\end{array}$ \\
\hline Adult Memory and Information Processing Battery & $\begin{array}{l}\text { Spatial Recall Test: Visuospatial memory } \\
\text { Symbol Digit Modalities Test: Concentration, psychomotor speed, and graphomotor abilities } \\
\text { Paced Auditory Serial Addition Test: Auditory information processing speed and working memory } \\
\text { Word Generation List: Lexical fluency Selective Reminding Test: Verbal learning and memory }\end{array}$ \\
\hline Brief Neurocognitive Battery & $\begin{array}{l}\text { Animal Fluency: Semantic fluency and executive control } \\
\text { Boston Naming Test-15: Expressive language } \\
\text { Coding: Attention and visuomotor processing } \\
\text { Digit Span: Auditory attention and working memory } \\
\text { Stroop Color Naming: Attention and speed of information processing } \\
\text { Stroop Word Reading: Attention and speed of word reading } \\
\text { Stroop Interference: Inhibition and cognitive flexibility } \\
\text { Trails Making Test-A: Simple attention, visual scanning and processing speed } \\
\text { Trails Making Test-B: Visual scanning, divided attention and cognitive flexibility }\end{array}$ \\
\hline
\end{tabular}

of the THC dose inhaled (39-45). Importantly, none of the studies collected blood to measure plasma levels of THC and its metabolites. It would have been informative to have been able to directly relate objectively measured cognitive impairment across specific domains to plasma levels of cannabinoids in these subjects.

Several observations from this review draw important comparisons with the recreational cannabis literature. As we have already discussed in detail the results of the scoping review and the seven studies in the Summary of Findings above, the focus on the present Discussion is to highlight and discuss important considerations when reviewing the current literature in addition to a variety of modifiable and non-modifiable factors that were found to influence the duration and degree of neurocognitive impairment in medical cannabis patients (see Figure 2).

There are several non-modifiable factors, intrinsic to the patient, that influence both the degree and duration of impairment (Figures 2A-C). These important factors are sometimes overlooked within the larger body of literature, particularly within recreational studies.

\section{Genetics and Metabolism}

Genetic and metabolic profiles or predispositions influence how an individual responds to cannabis, and thus the side effects experienced. Genetics, such as variations in the COMT/AKT genotype $(46,47)$, individual endocannabinoid system "tone" [endogenous endocannabinoid levels, receptor sensitivity and abundance, which may be altered in psychiatric conditions such as depression $(48,49)]$, as well as hypo- or hypermetabolizers can influence how THC is metabolized (50) and thus the degree and duration of impairment experienced by an individual
(Figure 2B). This may influence study outcomes, particularly when smaller sample sizes are used.

\section{Personal or Family Mental Health History}

It is important to consider personal or family mental health history when assessing factors of impairment. Experienced or known pre-dispositions to some mental health conditions may increase the risk of impairment for some individuals (Figure 2B) (51, 52). The use of high THC chemovars may exacerbate this risk.

\section{Comorbidities}

Studies that assess the therapeutic effects of THC based on ability to manage symptoms, predominantly pain or spasticity, should acknowledge that these symptoms may contribute to impairment (Figure 2C). Patients with certain medical conditions, such as multiple sclerosis, epilepsy, insomnia, anxiety, and depression, have twice the risk of motor vehicle accidents than healthy controls (53-55). Chronic pain syndromes can manifest with comorbid fatigue, weakness, dizziness, or cognitive slowing, which may compound the impairment produced by THC. However, by managing these symptoms with medical cannabis, baseline neurocognitive and psychomotor functioning may improve, as was reported in a driving simulation study with patients who have multiple sclerosis (56). Comorbidities with additive impairing effects should be carefully considered clinically and in future research. In addition to non-modifiable factors, this review identified several modifiable factors that were found to influence the duration and degree of impairment. These are now discussed in more detail below (Figures 2D-K). 


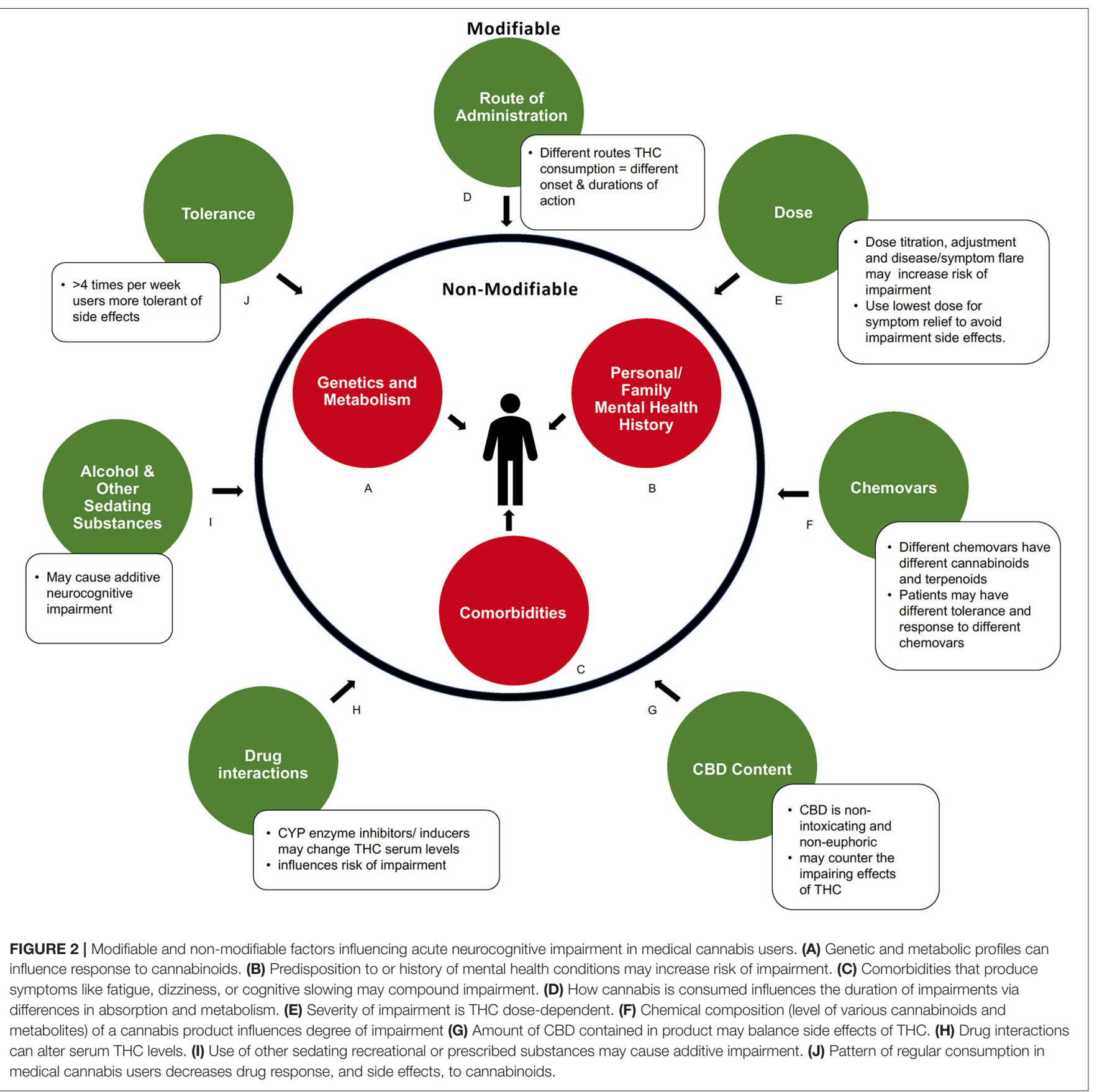

\section{Route of Administration}

As represented in Figure 2D, there is a clear difference in the duration of neurocognitive impairment depending on the route of administration (smoked vs. sublingual spray vs. oils). Due to differences in absorption and metabolism, THC has a different onset and duration of action depending on where in the body it is administered (57-59). Cannabis oils may provide up to $8 \mathrm{~h}$ of symptom relief due to gradual absorption of THC from the gut combined with first pass metabolism conversion of THC to 11-OH-THC, another active compound, in the liver $(30,58)$. The longer duration of therapeutic action also gives ingested formulations a greater period of potential impairment. Inhaled or vaporized THC produces a shorter period of impairment compared to oral formulations, with typical onset with 5$10 \mathrm{~min}$ and duration for $3-4 \mathrm{~h}$. This is due to rapid absorption of THC from the lungs into the bloodstream, with minimal conversion to 11-OH-THC by the liver via first-pass metabolism $(30,60-62)$. Although none of the studies above utilized oil ingestible THC formulations, clinically this is a common method of intake for patients using medical cannabis, to limit the negative effects of smoking. We would recommend that future studies administer cannabis oils, providing doses similar to those that 
are prescribed in practice, in order to appropriately represent the medical cannabis population. Further, new formulations are being manufactured with different carrier oils, extraction techniques, and cannabinoid content which may lead to different levels and duration of impairment. Future pharmacokinetic studies assessing these formulations are needed.

\section{Dose}

The degree and duration of neurocognitive impairment is dose-dependent, with higher THC doses being more impairing than lower doses. The dose of THC used among the medical cannabis studies reviewed were substantially lower compared to typical recreational studies (Figure 2E). Recreational studies often measure neurocognitive functioning in heavy cannabis users and follow the participants usual cannabis regimen, with a reported average of two cannabis "joints" per dosing session (63-66). If one "joint" contains $\sim 750 \mathrm{mg}$ of cannabis with a THC concentration of $15 \%$, one dosing session would contain $225 \mathrm{mg}$ of THC. Some of these high-dose THC recreational studies have shown subtle defects in cognitive tasks up to 24-h after THC inhalation (65). However, recreational studies using doses similar to this medical cannabis review, [with the highest dose administered being $34 \mathrm{mg}$ of THC (40)], do not note any neurocognitive impairment 24-h after THC ingestion (67).

Rather than using data from studies with medical cannabis users and with doses typically used by medical cannabis patients, Health Canada's "Cannabis Impairment" report based its conclusions on data from studies of recreational cannabis, where doses are substantially higher. The report notes: "(s)ome effects of cannabis use, for example drowsiness, can last up to $24 \mathrm{~h}$, well after other effects may have faded...(T)here is no standard waiting time to drive after using cannabis. If you are using cannabis, do not drive." (68). If they followed these recommendations, many daily medical cannabis patients would be unable to drive or attend work, even if they only utilize THC at night before going to sleep.

This review of the literature found no reports of neurocognitive deficits with THC use 4-h after inhalation using modest THC-dosing strategies. We would recommend using lower-THC doses, (as were seen in the studies in this review), for daily symptom management, as higher doses may prolong the duration of impairment.

\section{Chemovars and CBD Content}

The addition of other cannabinoids, such as CBD, may have an impact on the severity of neurocognitive impairment (Figures 2F,G) (69). One of the studies in this review, compared oromucosal spray formulations of THC vs. THC: CBD 1:1 vs. $\mathrm{CBD}$ vs. placebo and noted that participants in the THC: CBD group had less drowsiness, dysphoria, and euphoria (Figure 2F) (42). In addition to CBD, cannabis contains many other cannabinoids and terpenes that may affect neurocognitive impairment (Figure 2F). For example, myrcene may potentiate the sedating effects of THC $(70,71)$. Importantly, this could mean that patients who develop tolerance to the unwanted neurocognitive side effects of one chemovar of cannabis may not have the same tolerance to other chemovars with different concentrations of cannabinoids and terpenoids (70). Thus, another informative avenue for future studies would be to monitor and record in detail the quantities and concentrations of the other constituents of the cannabis being studied, as the individual or "entourage" effects of these on cognitive impairment is largely unknown.

\section{Drug Interactions and Sedating Substances}

Medical cannabis patients often utilize other impairing substances to manage their conditions. The interaction of these substances with THC may further the duration and severity of neurocognitive impairment (Figures 2H,I). For example, there is the potential for additive impairment due to interactions with other intoxicants (e.g., alcohol) or sedating medications such as benzodiazepines, opioids, tricyclic antidepressants, and anti-epileptics (Figure 2I) (58). All studies in the current review required patients to stay on their normal routine medications (39-45), and only one study excluded participants who were on opioid medications or used any other medication deemed to interact with cannabis (45). The articles in this review did not list which medications were routinely consumed by patients, which would have been useful information. Most of the articles provide a brief summary of the major medical conditions that were associated with medical cannabis use, so some inferences can be drawn, but detailed information is missing. In clinical practice, it has been commonly noted that many patients reduce their use of prescription medications if they achieve greater symptom relief with marijuana, which can actually reduce overall sedation. Further, polypharmacy may result in drug interactions (Figure 2H). THC is metabolized by the CYP family of enzymes, therefore, CYP inducers or inhibitors may alter serum levels of THC, influencing risk of impairment $(58,72)$. It will therefore be important for future studies to report any relevant patient medications as potential confounding factors.

\section{Tolerance}

One of the important differences between the medical cannabis patient and those who use recreational cannabis is the pattern of THC use (e.g., intermittent vs. daily consumption). Medical cannabis patients typically manage symptoms using THC on a daily basis, which can lead to pharmacological tolerance, including tolerance to possible side effects (Figure 2J) (73-77). For example, a study of patients with multiple sclerosis did not demonstrate impairment in driving-related tasks after 4-6 weeks of daily medical cannabis treatment (when compared to their baseline without medical cannabis) (78). Notably, the one study where all participants used their daily medical cannabis up until testing day found improved performance compared to normative data (45). This suggests that patients who take medical cannabis every day may not develop the same amount of neurocognitive impairment as those who previously abstained or use infrequently.

Some of the studies evaluated in this review enrolled participants with a previous history of cannabis use $(44,45)$, while others enrolled cannabis naïve participants $(41,43)$, which may contribute to the significant heterogeneity between study results. 
Even within medical cannabis patients, those who use medical cannabis for persistent, chronic daily symptoms vary significantly in their use patterns from those who use to control acute and intermittent symptoms. Future clinical studies should consider THC tolerance and ensure that the duration and amount of previous THC use is specified in the eligibility criteria and evaluated when interpreting results. A standardized definition for chronic, daily medical cannabis use should be implemented in future studies. For most patients, titration and monitoring of cannabis intake typically takes $4-12$ weeks to achieve an optimal therapeutic effect. The titration period depends on a number of factors (Figures 2A-C,I) including comorbidities, polypharmacy, genetics, and age (30). A research definition should account for this titration period and consider stabilization to have occurred when no further dose adjustments are required over a 2 week period. This will ultimately increase the validity and applicability to research findings. Further reviews and commentary on factors that influence impairment (Figure 2) are greatly needed.

\section{Limitations}

Findings from this review were constrained by the limitations of the current literature. Due to the heterogeneity of the study populations, study designs and protocols, and variability in the objective testing measures between studies, we were unable to complete a meta-analysis. The lack of cognitive and motor test standardization and the inconsistent methods between studies, including the type and time of testing post-THC ingestion, precluded statistical pooling of the data. There were no standardized medical cannabis products used across studies, with each study exploring varying concentrations of THC and CBD in either smoked, vaporized, or sublingual formulations, including cannabis-based medicines such as THC:CBD oromucosal spray (Figures 2F,G). Combining findings between the included studies and coming to definitive conclusions would be premature.

An additional limitation in the literature was lack of research assessing oral THC products, including cannabis oils. Due to the known pharmacokinetic differences between ingested and inhaled THC and given that many medical cannabis patients use oral formulations, it will be important for future studies to incorporate these products in their trials. An important confounder in studies on impairment are the participants underlying medical conditions (which in these studies often included illnesses that are detrimental to neurocognitive performance). Patients baseline cognitive functioning was only described and controlled for in three of the six studies $(39,40,43)$, and is important to document for future studies. Blood levels of THC and its metabolites were also not assessed in any of these studies. This was a missed opportunity to obtain a better understanding of how drug levels relate to cognitive impairment in medical cannabis users with medical doses. It would also have better enabled comparison of effects between medical and recreational cannabis users.

Finally, the literature on this topic is limited by the relatively small sample sizes of included studies. Small sample sizes
TABLE 5 | Summary of findings.

\section{Summary of findings}

Neurocognitive impairment following cannabis inhalation is less than or equal to $4 \mathrm{~h}$ in medical cannabis patients, independent of their dosing regimen (e.g., daily, intermittent, or infrequent)

Impairment is THC dose-dependent

Acute impairment was found to be statistically significant in the following neurocognitive and psychomotor domains:

- Immediate and delayed verbal recall

- Processing speed

- Task switching

- Visual attention

- Fine motor coordination

- Working memory

There are several non-modifiable factors that influence duration and degree of impairment:

- Comorbidities

- Personal/ Family Mental Health History

- Genetics and metabolism

Medical cannabis patients consume cannabis to manage symptoms and improve quality of life by optimizing the following modifiable domains:

- Intent of use

- Route of administration

- Chemovar selection

- CBD content

- Dose

- Tolerance

- Alcohol \& other sedating substances

- Drug interactions

We cannot extrapolate the conclusions found in this review to recreational cannabis populations or those "medical cannabis" patients not under the guidance of a health care practitioner.

may overestimate treatment effects or be insufficiently powered to detect a true difference, although some studies stated they were sufficiently powered to detect differences. Future trials would provide more robust information if they had larger sample sizes and captured data on a wider range of medical cannabis patients. Nevertheless, the trends that emerged among these medical cannabis impairment studies compared to the recreational data supports that medical cannabis patients do not have the same duration or degree of neurocognitive impairment as recreational users.

\section{CONCLUSIONS}

This review suggests that the duration of neurocognitive impairment following inhalation or sublingual absorption of THC containing products is $4 \mathrm{~h}$ or less in medical cannabis patients. The results of this review are consistent with the College of Family Physicians of Canada's 2014 statement that medical cannabis patients should err on the side of caution, and delay safety sensitive activities for $3-4 \mathrm{~h}$ if cannabis (THC) is inhaled, $6-8 \mathrm{~h}$ if ingested orally, and $8 \mathrm{~h}$ if any euphoria is experienced (79). There are important differences between medical and recreational cannabis users that may not allow for the same conclusions to be drawn about the duration or degree 
of impairment within the recreational cannabis population. These differences pertain to factors including the dose of THC, method of intake, patient tolerance and intent, additional chemovars added (such as CBD) and concurrent sedative or hypnotic medication intake (Figure 2). This review suggests that neurocognitive impairment in medical cannabis patients can involve multiple neurocognitive and psychomotor domains. A summary of the main conclusions and recommendations from this review can be found in Table 5 .

\section{DATA AVAILABILITY STATEMENT}

The original contributions presented in the study are included in the article/supplementary material, further inquiries can be directed to the corresponding author.

\section{REFERENCES}

1. Rouhollahi E, MacLeod BA, Barr AM, Puil E. Cannabis extract CT-921 has a high efficacy-adverse effect profile in a neuropathic pain model. Drug Des Deve Ther. (2020) 14:3351-61. doi: 10.2147/DDDT.S247584

2. Gonçalves J, Rosado T, Soares S, Simão AY, Caramelo D, Luís Â, et al. Cannabis and its secondary metabolites: their use as therapeutic drugs, toxicological aspects, and analytical determination. Medicines (Basel, Switzerland). (2019) 6:31. doi: 10.3390/medicines6010031

3. Alves P, Amaral C, Teixeira N, Correia-da-Silva G. Cannabis sativa: much more beyond $\Delta(9)$-tetrahydrocannabinol. Pharmacol Res. (2020) 157:104822. doi: 10.1016/j.phrs.2020.104822

4. Yau JC, Yu SM, Panenka WJ, Pearce H, Gicas KM, Procyshyn RM, et al. Characterization of mental health in cannabis dispensary users, using structured clinical interviews and standardized assessment instruments. BMC Psychiatry. (2019) 19:335. doi: 10.1186/s12888-019-2324-z

5. Geoffrion R, Yang EC, Koenig NA, Brotto LA, Barr AM, Lee T, et al. Recreational cannabis use before and after legalization in women with pelvic pain. Obstet Gynecol. (2020) 137:91-9. doi: 10.1097/AOG.0000000000004207

6. Moulin D, Boulanger A, Clark AJ, Clarke H, Dao T, Finley GA, et al. Pharmacological management of chronic neuropathic pain: revised consensus statement from the Canadian Pain Society. Pain Res Manag. (2014) 19:32835. doi: 10.1155/2014/754693

7. Lynch ME, Ware MA. Cannabinoids for the treatment of chronic non-cancer pain: an updated systematic review of randomized controlled trials. J Neuroimmune Pharmacol. (2015) 10:293-301. doi: 10.1007/s11481-015-9600-6

8. Abrams DI, Couey P, Shade SB, Kelly ME, Benowitz NL. Cannabinoidopioid interaction in chronic pain. Clin Pharmacol Ther. (2011) 90:84451. doi: 10.1038/clpt.2011.188

9. Nielsen S, Sabioni P, Trigo JM, Ware MA, Betz-Stablein BD, Murnion B, et al. Opioid-sparing effect of cannabinoids: a systematic review and meta-analysis. Neuropsychopharmacology. (2017) 42:1752-65. doi: 10.1038/npp.2017.51

10. Boehnke KF, Litinas E, Clauw DJ. Medical cannabis use is associated with decreased opiate medication use in a retrospective crosssectional survey of patients with chronic pain. J Pain. (2016) 17:739-44. doi: 10.1016/j.jpain.2016.03.002

11. Hurd YL. Cannabidiol: swinging the marijuana pendulum from 'weed' to medication to treat the opioid epidemic. Trends Neurosci. (2017) 40:1247. doi: $10.1016 /$ j.tins.2016.12.006

12. Weil AT. Marihuana. Science. (1969) 163:1145. doi: 10.1126/science.163.3872.1145

13. Herkenham M, Lynn AB, Little MD, Johnson MR, Melvin LS, de Costa BR, et al. Cannabinoid receptor localization in brain. Proc Natl Acad Sci U S A. (1990) 87:1932-6. doi: 10.1073/pnas.87.5.1932

14. Glass M, Dragunow M, Faull RL. Cannabinoid receptors in the human brain: a detailed anatomical and quantitative autoradiographic study in

\section{AUTHOR CONTRIBUTIONS}

LE was primarily responsible for the review of published abstracts, with additional support from LL, and wrote the first draft. CM supervised the project and provided the overall intellectual leadership. All other authors contributed to revising the manuscript with additional intellectual input.

\section{ACKNOWLEDGMENTS}

We would like to acknowledge Michael Boivin for his expertise in cannabis knowledge translation, and Dr. Zachary Walsh and Michelle St. Pierre for their suggestions on earlier drafts of this review.

the fetal, neonatal and adult human brain. Neuroscience. (1997) 77:299318. doi: 10.1016/S0306-4522(96)00428-9

15. Brubacher JR, Chan H, Staples JA. Cannabis-impaired driving and Canadian youth. Paediatr Child Health. (2020) 25(Suppl.1):S21s5. doi: $10.1093 / \mathrm{pch} / \mathrm{pxaa} 017$

16. Busardò FP, Pellegrini M, Klein J, di Luca NM. Neurocognitive correlates in driving under the influence of cannabis. CNS Neurol Disord Drug Targets. (2017) 16:534-40. doi: 10.2174/1871527316666170424115455

17. Busardo FP, Pichini S, Pellegrini M, Montana A, Lo Faro AF, Zaami S, et al. Correlation between blood and oral fluid psychoactive drug concentrations and cognitive impairment in driving under the influence of drugs. Curr Neuropharmacol. (2018) 16:84-96. doi: 10.2174/1570159X15666170828162057

18. Ramaekers JG, Kauert G, van Ruitenbeek P, Theunissen EL, Schneider E, Moeller MR. High-potency marijuana impairs executive function and inhibitory motor control. Neuropsychopharmacology. (2006) 31:2296303. doi: 10.1038/sj.npp.1301068

19. Bramness JG, Khiabani HZ, Mørland J. Impairment due to cannabis and ethanol: clinical signs and additive effects. Addiction. (2010) 105:10807. doi: 10.1111/j.1360-0443.2010.02911.x

20. Ramaekers JG, Moeller MR, van Ruitenbeek P, Theunissen EL, Schneider E, Kauert G. Cognition and motor control as a function of Delta9THC concentration in serum and oral fluid: limits of impairment. Drug Alcohol Depend. (2006) 85:114-22. doi: 10.1016/j.drugalcdep.2006. 03.015

21. Compton R. Marijuana-Impaired Driving-A Report to Congress. Washington, DC: Administration NHTS (2017).

22. Grotenhermen F. Pharmacokinetics and pharmacodynamics of cannabinoids. Clin Pharmacokinet. (2003) 42:32760. doi: 10.2165/00003088-200342040-00003

23. Huestis MA. Human cannabinoid pharmacokinetics. Chem Biodivers. (2007) 4:1770-804. doi: 10.1002/cbdv.200790152

24. Smith-Kielland A, Skuterud B, Mørland J. Urinary excretion of 11-nor-9-carboxy-delta9-tetrahydrocannabinol and cannabinoids in frequent and infrequent drug users. J Anal Toxicol. (1999) 23:323-32. doi: 10.1093/jat/23.5.323

25. Grotenhermen F. The toxicology of cannabis and cannabis prohibition. Chem Biodivers. (2007) 4:1744-69. doi: 10.1002/cbdv.200790151

26. Ramaekers JG, Kauert G, Theunissen EL, Toennes SW, Moeller MR. Neurocognitive performance during acute THC intoxication in heavy and occasional cannabis users. J Psychopharmacol. (2009) 23:266-77. doi: 10.1177/0269881108092393

27. Desrosiers NA, Ramaekers JG, Chauchard E, Gorelick DA, Huestis MA. Smoked cannabis' psychomotor and neurocognitive effects in occasional and frequent smokers. J Anal Toxicol. (2015) 39:251-61. doi: 10.1093/jat/ bkv012 
28. Broyd SJ, van Hell HH, Beale C, Yücel M, Solowij N. Acute and chronic effects of cannabinoids on human cognition-A systematic review. Biol Psychiatry. (2016) 79:557-67. doi: 10.1016/j.biopsych.2015.12.002

29. Turna J, Balodis I, Munn C, Van Ameringen M, Busse J, MacKillop J. Overlapping patterns of recreational and medical cannabis use in a large community sample of cannabis users. Compr Psychiatry. (2020) 102:152188. doi: 10.1016/j.comppsych.2020.152188

30. MacCallum CA, Russo EB. Practical considerations in medical cannabis administration and dosing. Eur J Intern Med. (2018) 49:12-9. doi: 10.1016/j.ejim.2018.01.004

31. Jones AA, Gicas KM, Seyedin S, Willi TS, Leonova O, Vila-Rodriguez F, et al. Associations of substance use, psychosis, and mortality among people living in precarious housing or homelessness: a longitudinal, community-based study in Vancouver, Canada. PLoS Med. (2020) 17:e1003172. doi: 10.1371/journal.pmed.1003172

32. Gicas KM, Cheng A, Panenka WJ, Kim DD, Yau JC, Procyshyn RM, et al. Differential effects of cannabis exposure during early versus later adolescence on the expression of psychosis in homeless and precariously housed adults. Prog Neuropsychopharmacol Biol Psychiatry. (2020) 106:110084. doi: 10.1016/j.pnpbp.2020.110084

33. Barbic SP, Jones AA, Woodward M, Piercy M, Mathias S, VilaRodriguez F, et al. Clinical and functional characteristics of young adults living in single room occupancy housing: preliminary findings from a 10-year longitudinal study. Can J Public Health. (2018) 109:204-14. doi: 10.17269/s41997-018-0087-9

34. Willi TS, Honer WG, Thornton AE, Gicas K, Procyshyn RM, Vila-Rodriguez F, et al. Factors affecting severity of positive and negative symptoms of psychosis in a polysubstance using population with psychostimulant dependence. Psychiatry Res. (2016) 240:336-42. doi: 10.1016/j.psychres.2016.04.059

35. Knerich V, Jones AA, Seyedin S, Siu C, Dinh L, Mostafavi S, et al. Social and structural factors associated with substance use within the support network of adults living in precarious housing in a socially marginalized neighborhood of Vancouver, Canada. PLoS ONE. (2019) 14:e0222611. doi: 10.1371/journal.pone.0222611

36. Tse L, Schwarz SK, Bowering JB, Moore RL, Burns KD, Richford CM, et al. Pharmacological risk factors for delirium after cardiac surgery: a review. Curr Neuropharmacol. (2012) 10:181-96. doi: 10.2174/157015912803217332

37. Linton D, Barr AM, Honer WG, Procyshyn RM. Antipsychotic and psychostimulant drug combination therapy in attention deficit/hyperactivity and disruptive behavior disorders: a systematic review of efficacy and tolerability. Curr Psychiatry Rep. (2013) 15:355. doi: 10.1007/s11920-013-0355-6

38. Yin J, Barr AM, Ramos-Miguel A, Procyshyn RM. Antipsychotic induced dopamine supersensitivity psychosis: a comprehensive review. Curr Neuropharmacol. (2017) 15:17483. doi: 10.2174/1570159X14666160606093602

39. Wallace MS, Marcotte TD, Umlauf A, Gouaux B, Atkinson JH. Efficacy of inhaled cannabis on painful diabetic neuropathy. J Pain. (2015) 16:61627. doi: 10.1016/j.jpain.2015.03.008

40. Wilsey B, Marcotte T, Tsodikov A, Millman J, Bentley H, Gouaux B, et al. A randomized, placebo-controlled, crossover trial of cannabis cigarettes in neuropathic pain. J Pain. (2008) 9:506-21. doi: 10.1016/j.jpain.2007. 12.010

41. Corey-Bloom J, Wolfson T, Gamst A, Jin S, Marcotte TD, Bentley H, et al. Smoked cannabis for spasticity in multiple sclerosis: a randomized, placebocontrolled trial. CMAJ. (2012) 184:1143-50. doi: 10.1503/cmaj.110837

42. Notcutt W, Price M, Miller R, Newport S, Phillips C, Simmons S, et al. Initial experiences with medicinal extracts of cannabis for chronic pain: results from 34 'N of 1' studies. Anaesthesia. (2004) 59:44052. doi: $10.1111 / j .1365-2044.2004 .03674 . x$

43. Wilsey B, Marcotte TD, Deutsch R, Zhao H, Prasad H, Phan A. An exploratory human laboratory experiment evaluating vaporized cannabis in the treatment of neuropathic pain from spinal cord injury and disease. J Pain. (2016) 17:982-1000. doi: 10.1016/j.jpain.2016.05.010

44. Wilsey B, Marcotte T, Deutsch R, Gouaux B, Sakai S, Donaghe H. Low-dose vaporized cannabis significantly improves neuropathic pain. J Pain. (2013) 14:136-48. doi: 10.1016/j.jpain.2012.10.009
45. Olla P, Rykulski N, Hurtubise JL, Bartol S, Foote R, Cutler L, et al. Short-term effects of cannabis consumption on cognitive performance in medical cannabis patients. Appl Neuropsychol Adult. (2019):1-11. doi: 10.1080/23279095.2019.1681424

46. Karcher NR, Barch DM, Demers CH, Baranger DAA, Heath AC, Lynskey MT, et al. Genetic predisposition vs individual-specific processes in the association between psychotic-like experiences and cannabis use. JAMA Psychiatry. (2019) 76:87-94. doi: 10.1001/jamapsychiatry.2018.2546

47. Lorenzetti V, Solowij $N$, Yücel $M$. The role of cannabinoids in neuroanatomic alterations in cannabis users. Biol Psychiatry. (2016) 79:e17-31. doi: 10.1016/j.biopsych.2015.11.013

48. Russo EB. Clinical endocannabinoid deficiency reconsidered: current research supports the theory in migraine, fibromyalgia, irritable bowel, and other treatment-resistant syndromes. Cannabis Cannabinoid Res. (2016) 1:15465. doi: $10.1089 /$ can.2016.0009

49. Hill MN, Barr AM, Ho WS, Carrier EJ, Gorzalka BB, Hillard CJ. Electroconvulsive shock treatment differentially modulates cortical and subcortical endocannabinoid activity. J Neurochem. (2007) 103:47-56. doi: 10.1111/j.1471-4159.2007.04688.x

50. Lynch T, Price A. The effect of cytochrome P450 metabolism on drug response, interactions, and adverse effects. Am Fam Physician. (2007) 76:3916.

51. Moore TH, Zammit S, Lingford-Hughes A, Barnes TR, Jones PB, Burke $M$, et al. Cannabis use and risk of psychotic or affective mental health outcomes: a systematic review. Lancet. (2007) 370:31928. doi: 10.1016/S0140-6736(07)61162-3

52. Hudson A, Hudson P. Risk factors for cannabis-related mental health harms in older adults: a review. Clin Gerontol. (2020) 44:1-13. doi: 10.1080/07317115.2020.1808134

53. McGwin G Jr, Sims RV, Pulley L, Roseman JM. Relations among chronic medical conditions, medications, and automobile crashes in the elderly: a population-based case-control study. Am J Epidemiol. (2000) 152:42431. doi: 10.1093/aje/152.5.424

54. Sagberg F. Driver health and crash involvement: a case-control study. Accid Analysis Preve. (2006)38:28-34. doi: 10.1016/j.aap.2005.06.018

55. Walsh Z, Callaway R, Belle-Isle L, Capler R, Kay R, Lucas P, et al. Cannabis for therapeutic purposes: patient characteristics, access, and reasons for use. Int J Drug Policy. (2013) 24:511-6. doi: 10.1016/j.drugpo.2013. 08.010

56. Freidel M, Tiel-Wilck K, Schreiber H, Prechtl A, Essner U, Lang M. Drug-resistant MS spasticity treatment with Sativex( $($ ) add-on and driving ability. Acta neurologica Scandinavica. (2015) 131:9-16. doi: 10.1111/ane. 12287

57. Poyatos L, Pérez-Acevedo AP, Papaseit E, Pérez-Mañá C, Martin S, Hladun O, et al. Oral administration of cannabis and $\Delta$-9-tetrahydrocannabinol (THC) preparations: a systematic review. Medicina (Kaunas, Lithuania). (2020) 56:309. doi: 10.3390/medicina56060309

58. Lucas CJ, Galettis P, Schneider J. The pharmacokinetics and the pharmacodynamics of cannabinoids. Br J Clin Pharmacol. (2018) 84:2477-82. doi: 10.1111/bcp. 13710

59. Foster BC, Abramovici H, Harris CS. Cannabis and cannabinoids: kinetics and interactions. Am J Med. (2019) 132:126670. doi: 10.1016/j.amjmed.2019.05.017

60. Heishman SJ, Huestis MA, Henningfield JE, Cone EJ. Acute and residual effects of marijuana: profiles of plasma THC levels, physiological, subjective, and performance measures. Pharmacol Biochem Behav. (1990) 37:5615. doi: 10.1016/0091-3057(90)90028-G

61. Mattes RD, Shaw LM, Edling-Owens J, Engelman K, Elsohly MA. Bypassing the first-pass effect for the therapeutic use of cannabinoids. Pharmacol Biochem Behav. (1993) 44:745-7. doi: 10.1016/0091-3057(93)90194-X

62. McGilveray IJ. Pharmacokinetics of cannabinoids. Pain Res Manag. (2005) 10(Suppl.A):15a-22a. doi: 10.1155/2005/242516

63. McHale S, Hunt $\mathrm{N}$. Executive function deficits in shortterm abstinent cannabis users. Hum Psychopharmacol. (2008) 23:409-15. doi: 10.1002/hup.941

64. Solowij N, Stephens RS, Roffman RA, Babor T, Kadden R, Miller M, et al. Cognitive functioning of long-term heavy cannabis users seeking treatment. JAMA. (2002) 287:1123-31. doi: 10.1001/jama.287.9.1123 
65. Block RI, Ghoneim MM. Effects of chronic marijuana use on human cognition. Psychopharmacology (Berl). (1993) 110:21928. doi: 10.1007/BF02246977

66. Pope HG Jr, Gruber AJ, Hudson JI, Huestis MA, Yurgelun-Todd D. Neuropsychological performance in long-term cannabis users. Arch Gen Psychiatry. (2001) 58:909-15. doi: 10.1001/archpsyc.58.10.909

67. Kurzthaler I, Hummer M, Miller C, Sperner-Unterweger B, Günther V, Wechdorn $\mathrm{H}$, et al. Effect of cannabis use on cognitive functions and driving ability. J Clin Psychiatry. (1999) 60:395-9. doi: 10.4088/JCP.v60 n0609

68. Government of Canada. Cannabis Impairment Ottawa, ON2019. Available online at: https://www.canada.ca/en/services/health/campaigns/cannabis/ impairment.html (accessed December 17, 2019).

69. Russo EB. Cannabidiol claims and misconceptions. Trends Pharmacol Sci. (2017) 38:198-201. doi: 10.1016/j.tips.2016.12.004

70. Russo EB. Taming THC: potential cannabis synergy and phytocannabinoid-terpenoid entourage effects. Br J Pharmacol. (2011) 163:1344-64. doi: 10.1111/j.1476-5381.2011.01238.x

71. Lewis MA, Russo EB, Smith KM. Pharmacological foundations of cannabis chemovars. Planta Med. (2018) 84:225-33. doi: 10.1055/s-0043-12 2240

72. Antoniou T, Bodkin J, Ho JM. Drug interactions with cannabinoids. CMAJ. (2020) 192:E206. doi: 10.1503/cmaj.191097

73. Nurmikko TJ, Serpell MG, Hoggart B, Toomey PJ, Morlion BJ, Haines D. Sativex successfully treats neuropathic pain characterised by allodynia: a randomised, double-blind, placebo-controlled clinical trial. Pain. (2007) 133:210-20. doi: 10.1016/j.pain.2007.08.028

74. Kurzthaler I, Bodner T, Kemmler G, Entner T, Wissel J, Berger T, et al. The effect of nabilone on neuropsychological functions related to driving ability: an extended case series. Hum Psychopharmacol. (2005) 20:2913. doi: $10.1002 /$ hup. 688

75. Rog DJ, Nurmikko TJ, Friede T, Young CA. Randomized, controlled trial of cannabis-based medicine in central pain in multiple sclerosis. Neurology. (2005) 65:812-9. doi: 10.1212/01.wnl.0000176753.45410.8b

76. Vaney C, Heinzel-Gutenbrunner M, Jobin P, Tschopp F, Gattlen B, Hagen U, et al. Efficacy, safety and tolerability of an orally administered cannabis extract in the treatment of spasticity in patients with multiple sclerosis: a randomized, double-blind, placebo-controlled, crossover study.
Multiple Sclerosis (Houndmills, Basingstoke, England). (2004) 10:41724. doi: 10.1191/1352458504ms1048oa

77. Ware MA, Wang T, Shapiro S, Collet JP. Cannabis for the management of pain: assessment of safety study (COMPASS). J Pain. (2015) 16:123342. doi: 10.1016/j.jpain.2015.07.014

78. Rekand T. THC:CBD spray and MS spasticity symptoms: data from latest studies. Eur Neurol. (2014) 71(Suppl.1):4-9. doi: 10.1159/00035 7742

79. College of Family Physicians of Canada. Authorizing Dried Cannabis for Chronic Pain or Anxiety: Preliminary Guidance from the College of Family Physicians of Canada. Mississauga, ON: College of Family Physicians of Canada (2014).

Conflict of Interest: CM has received support for industry sponsored continuing medical education from Canopy, Medreleaf, Doja, Compass Cannabis Clinics. She has been on the scientific advisory board of Emerald Health Therapeutics, Shoppers Drug Mart, Strainprint and Resolve. She is the Medical Director of Greenleaf medical clinic, Vitality and Translational Life Sciences. She is on the Board of Directors for The Green Organic Dutchman. WP is the founder and CEO of Translational Life Sciences, an early stage science company focused on cannabinoid therapeutics. He has also been on the scientific advisory board of Medipure Pharmaceuticals and Vitality Biopharma, and in the past has been on the board of directors for Abbatis bioceuticals and on the advisory board of Vinergy Resources. $\mathrm{AB}$ has been a scientific advisor to Emerald Health Therapeutics, Cannevert Therapeutics, Global Cannabis Applications Corp, Medipure Pharmaceuticals, Vitality Biopharma and Hai Beverages.

The remaining authors declare that the research was conducted in the absence of any commercial or financial relationships that could be construed as a potential conflict of interest.

Copyright (C) 2021 Eadie, Lo, Christiansen, Brubacher, Barr, Panenka and MacCallum. This is an open-access article distributed under the terms of the Creative Commons Attribution License (CC BY). The use, distribution or reproduction in other forums is permitted, provided the original author(s) and the copyright owner(s) are credited and that the original publication in this journal is cited, in accordance with accepted academic practice. No use, distribution or reproduction is permitted which does not comply with these terms. 